Jahrbuch für Rechtssoziologie und Rechtstheorie V 


\section{Jahrbuch für Rechtssoziologie und Rechtstheorie}

Herausgegeben in Verbindung mit

Erich Fechner, Arthur Kaufmann, Ulrich Klug,

Niklas Luhmann, Peter Noll, Heinrich Popitz, Manfred Rehbinder, Rüdiger Schott, Paul Trappe

von Werner Maihofer und Helmut Schelsky

Band V 


\title{
Rechtsbedürfnis und Rechtshilfe
}

\author{
Empirische Ansätze im \\ internationalen Vergleich
}

Herausgegeben von

Erhard Blankenburg und Wolfgang Kaupen 
CIP-Kurztitelaufnahme der Deutschen Bibliothek

Jahrbuch für Rechtssoziologie und Rechtstheorie /

hrsg. in Verbindung mit Erich Fechner ... von

Werner Maihofer u. Helmut Schelsky. - Opladen:

Westdeutscher Verlag.

NE: Maihofer, Werner [Hrsg.]

Bd. 5. $\rightarrow$ Rechtsbedürfnis und Rechtshilfe

Rechtsbedürfnis und Rechtshilfe: empir.

Ansätze im internat. Vergl. / hrsg. von Erhard

Blankenburg $\mathbf{u}$. Wolfgang Kaupen. - Opladen:

Westdeutscher Verlag, 1978.

(Jahrbuch für Rechtssoziologie und Rechts-

theorie; Bd. 5)

ISBN 978-3-663-05388-0

ISBN 978-3-663-05387-3 (eBook)

DOI 10.1007/978-3-663-05387-3

NE: Blankenburg, Erhard [Hrsg.]

(C) 1978 Westdeutscher Verlag, Opladen

Softcover reprint of the hardcover 1st edition 1978

Satz: Vieweg, Wiesbaden

Alle Rechte vorbehalten. Auch die fotomechanische Vervielfältigung des Werkes (Fotokopie, Mikrokopie) oder von Teilen daraus bedarf der vorherigen Zustimmung des Verlages. 


\section{Inhalt}

Einführung der Herausgeber $\ldots \ldots \ldots \ldots \ldots \ldots \ldots \ldots \ldots$

Josef Falke: Zugang zum Recht - Eine Fallstudie über die Öffentliche Rechtsauskunfts- und Vergleichsstelle in Hamburg. . . . . . . . . 13

Udo Reifner: Unentgeltliche Rechtsberatung in West-Berlin . . . . . . . 43

Götz von Aulock: Rechtshilfe für untere Schichten und Anwaltschaft . . . . . 94

Kees Schuyt / Kees Groenendijk / Bernard Sloot: Rechtsprobleme oder private Schwierigkeiten - Die Inanspruchnahme von Rechtshilfe in den Niederlanden . . . . . . . . . . . . . . . . . . . . . 109

Richard H. H. White: Rechtshilfe in England . . . . . . . . . . . 139

Martin Partington: Die Entwicklung der Rechtshilfe in England - aktuelle Reformbestrebungen . . . . . . . . . . . . . . . 150

Amedeo Cottino / Ulf Drugge / Eva Christina Johansson / Gudrun Nordborg: Die schwedische Rechtshilfereform . . . . . . . . . . . . 162

Jon T. Johnsen: Rechtshilfe in Norwegen . . . . . . . . . . . 175

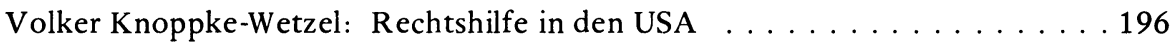

Erhard Blankenburg: Rechtshilfebedürfnis und Rechtsberatung Theoretische Überlegungen zur rechtspolitischen Diskussion in der Bundesrepublik Deutschland $\ldots \ldots \ldots \ldots \ldots \ldots \ldots 231$

Bibliographie . . . . . . . . . . . . . . . . . 250 